\title{
A role for the basal ganglia in nicotinic modulation of the blink reflex
}

\author{
Craig Evinger ${ }^{1.2}$, Michele A. Basso ${ }^{4}$, Karen A. Manning ${ }^{2}$, Patrick A. Sibony ${ }^{3}$, \\ John J. Pellegrini ${ }^{1}$, and Anja K.E. Horn ${ }^{1}$ \\ ${ }^{1}$ Department of Neurobiology and Behavior, ${ }^{3}$ Department of Ophthalmology, and ${ }^{4}$ Department of Psychology, \\ State University of New York at Stony Brook, Stony Brook, NY 11794, USA \\ ${ }^{2}$ Department of Anatomy, Medical School, University of Wisconsin, Madison, WI 53706, USA
}

Received December 6, 1991 / Accepted June 23, 1992

\begin{abstract}
Summary. In humans and rats we found that nicotine transiently modifies the blink reflex. For blinks elicited by stimulation of the supraorbital branch of the trigeminal nerve, nicotine decreased the magnitude of the orbicularis oculi electromyogram (OOemg) and increased the latency of only the long-latency (R2) component. For blinks elicited by electrical stimulation of the cornea, nicotine decreased the magnitude and increased the latency of the single component of OOemg response. Since nicotine modified only one component of the supraorbitally elicited blink reflex, nicotine must act primarily on the central nervous system rather than at the muscle. The effects of nicotine could be caused by direct action on lower brainstem interneurons or indirectly by modulating descending systems impinging on blink interneurons. Since precollicular decerebration eliminated nicotine's effects on the blink reflex, nicotine must act through descending systems. Three lines of evidence suggest that nicotine affects the blink reflex through the basal ganglia by causing dopamine release in the striatum. First, stimulation of the substantia nigra mimicked the effects of nicotine on the blink reflex. Second, haloperidol, a dopamine $\left(D_{2}\right)$ receptor antagonist, blocked the effect of nicotine on the blink reflex. Third, apomorphine, a $\mathrm{D}_{2}$ receptor agonist, mimicked the effects of nicotine on the blink reflex.
\end{abstract}

Key words: Blink reflex - Nicotine - Basal ganglia Orbicularis oculi - Rat - Human

\section{Introduction}

Cigarette smoking transiently modifies the human blink reflex elicited by stimulation of the supraorbital branch of the trigeminal nerve (SO; Evinger et al. 1988). Immediately after a person smokes a cigarette, the electromyogram (EMG) amplitude of the lid-closing muscle, the orbicularis

Correspondence to: $\mathrm{C}$. Evinger oculi decreases and the latency of the late R2 EMG component increases. In contrast, the latency of the early R1 EMG component does not change, although the EMG amplitude of both components decreases. The blink reflex returns to normal 3-5 min later. Two lines of evidence suggest that nicotine is the agent in cigarette smoke responsible for modifying the blink reflex. First, the time course of the rise and fall of blood nicotine levels following cigarette smoking (Benowitz et al. 1989; Russell et al. 1980, 1983) is similar to the time course of blink modifications with smoking. Second, nicotine is known to cross the blood-brain barrier readily. The present paper confirms that nicotine inhaled in cigarette smoke modifies the blink reflex (Evinger et al. 1988) by demonstrating that nicotine chewing gum induces the same modification of the blink reflex in humans as does cigarette smoking. Moreover, intravenous injection of nicotine in anesthetized rats modifies corneally and air-puff-elicited blinks in a similar manner to cigarette smoking in humans.

Nicotine could alter the blink reflex directly, by binding to nicotinic receptors on neurons that are part of the posterior brainstem blink circuits, or indirectly, by activating descending pathways that modulate the blink reflex. We eliminated the possibility of direct nicotinic effects by demonstrating that nicotine no longer modifies reflex blinking in decerebrate rats. The basal ganglia is an ideal candidate to account for an indirect effect of nicotine. First, basal ganglia disorders modify the blink reflex. Patients with Huntington's chorea, in which striatal dopamine levels are relatively high, exhibit a decrease in blink reflex excitability and a small increase in blink latency (Bollen et al. 1986; Carenceni et al. 1976; Esteban et al. 1981; Ferguson et al. 1978). In contrast, patients with Parkinson's disease, in which the striatal dopamine levels are abnormally low, possess a hyperexcitable blink reflex (Klawans and Goodwin 1969; Messina et al. 1972; Pearce et al. 1969; Rushworth 1962). Second, the basal ganglia are rich in nicotinic receptors, particularly on dopaminecontaining presynaptic terminals in the striatum (Araujo et al. 1988; Bolam et al. 1991; Clarke 1989; Hunt and Schmidt 1978; Semba and Fibiger 1989). Activation of 
these nicotinic receptors in the striatum and substantia nigra causes a release of dopamine (Clarke et al. 1985; Giorguieff-Chesselet et al. 1979; Lichtensteiger et al. 1982; Mereu et al. 1987; Sakurai et al. 1982; Schultz and Zigmond 1989; Westfall et al. 1989). These observations suggest that nicotine could modulate the blink reflex by causing a surge in dopamine release in the striatum. The present results confirm this hypothesis, by demonstrating that a dopamine $\left(\mathrm{D}_{2}\right)$ receptor antagonist blocks the effect of nicotine on the blink reflex and that a $\mathrm{D}_{2}$ receptor agonist mimics nicotine's effects. Thus, nicotine offers a tool for investigating how the basal ganglia may modulate reflexes in normal and diseased states.

\section{Materials and methods}

\section{Human experiments}

We investigated the reflex blinks of one man before and after chewing nicotine gum. The subject was aware of the goals of the investigation. All procedures were carried out in accordance with the SUNY Stony Brook Human Subject Guidelines.

\section{Preparation}

Evinger et al. (1991) have given a complete description of the measurement techniques for lid position and orbicularis oculi EMG (OOemg) activity in humans. Briefiy, we used the magnetic search coil technique to measure lid position by taping a 4-mm-diameter coil on the lower margin of the upper eyelid and then placing the subject in a magnetic field (C-N-C Enterprises). To calibrate the output of the coil, the subject fixated different points along the vertical meridian while the investigator measured the angle of lid rotation with a protractor. Two miniature silver electrodes $(<2 \mathrm{~mm}$ diameter) taped to the lateral and medial portions of the upper eyelid monitored OOemg, and a gold cup electrode ( $9 \mathrm{~mm}$ diameter, Grass) served as a ground. Neither the OOemg electrodes nor the coil impaired lid movement or caused discomfort to the subject. To elicit reflex blinks, two additional Grass skin electrodes were taped over the SO, one on the brow and another $1 \mathrm{~cm}$ above the first. Prior to electrode application, all skin surfaces were cleaned with alcohol and the electrodes coated with a conductive paste.

Prior to chewing nicotine gum (2 mg nicotine per tablet; Nicorette, Lakeside Pharmaceutical), we recorded 15 reflex blinks in response to electrical stimulation of the SO. The subject then chewed $10 \mathrm{mg}$ of nicotine gum for $3 \mathrm{~min}$. This was followed by 20 more electrical stimuli to the SO, identical to those delivered prior to chewing the nicotine in gum. Electrical stimuli were delivered every $20 \pm 3$ s. Data were collected and stored on-line on a personal computer. Lid and OOemg were sampled at $5000 \mathrm{~Hz}$, the computer rectified the OOemg record and integrated the muscle activity. The records could be analyzed individually or averaged (Evinger et al. 1991).

\section{Rat experiments}

Reflex blinks were also elicited in 11 male Sprague-Dawley rats weighing between 200 and $700 \mathrm{~g}$. All animal experiments were performed with the approval of and in accordance with SUNY Stony Brook Institutional Animal Care and Use Committee, state, and federal guidelines.

\section{Anesthetized rats}

We anesthetized rats with i.p. injections of urethane $(15 \mathrm{~g} / \mathrm{kg}$ in phosphate buffer $\mathrm{pH}$ 7.4). The femoral vein was cannulated to allow injection of nicotine. After fixing the head in a stereotaxic apparatus, we implanted a single-stranded, Teflon-coated, stainless steel wire [A-M Systems 7910; bare diameter $0.0762 \mathrm{~mm}$ diameter with Teflon coating $0.1143 \mathrm{~mm}$ ], bared for $1 \mathrm{~mm}$ at the tip, into the upper eyelid and a second into the lower eyelid of the rat to measure OOemg. Reflex blinks were elicited by electrical stimulation of the cornea with two small, silver balls or with brief air puffs directed at the eye.

In three rats, we tested the effect of nicotine injection alone on the blink reflex. Prior to i.v. injection of nicotine, 15 or 20 reflex blinks were elicited by electrical stimulation of the cornea or with an air puff presented every $20 \pm 3 \mathrm{~s}$. With this time between stimuli, blinks were consistent and robust. The rat was injected with nicotine, the amounts varying from $0.32 \mathrm{mg} / \mathrm{kg}$ to $2.5 \mathrm{mg} / \mathrm{kg}$ depending upon the experiment, and then received 20-40 stimuli identical to those presented prior to the nicotine injection. Two hours after completion of the first nicotine injection we repeated the procedure on each rat, using the same dose of nicotine, with similar results. At the end of the experiment, the rat was perfused with warm $6 \%$ dextran in phosphate buffer $(\mathrm{pH} 7.4)$ followed by cold $4 \%$ paraformaldehyde.

To show that rostral brain regions were not critical for the nicotine effect on blinking, the nicotine effect on blinking was demonstrated on two rats and then the rats were decerebrated precollicularly by aspiration. Two hours later, the effect of nicotine on the blink reflex was retested.

To examine whether basal ganglia activation could mimic the effect of nicotine, a stimulating microelectrode was implanted stereotaxically in the substantia nigra of two urethane-anesthetized rats. Twenty trials of OOemg activity evoked by stimulation of the cornea were performed. Data from 20 more trials using identical corneal stimuli were collected, but the substantia nigra region received a $10-\mathrm{Hz}, 40-\mu \mathrm{A}, 60-\mu \mathrm{s}$ stimulation for $7 \mathrm{~s}$ immediately preceding, but not overlapping, each corneal stimulus. Substantia nigra stimulation alone never produced any OOemg activity. The response to 20 additional corneal stimuli without nigral stimulation were then collected to show that the unstimulated blink reflex remained unchanged. The location of the stimulating electrodes was verified histologically for each experiment.

To evaluate the importance of dopamine release in nicotine's modulation of the blink reflex in two urethane-anesthetized rats, the effects of i.v. injections of nicotine were demonstrated and then the rats received i.p. injections of haloperidol $(0.15 \mathrm{mg} / \mathrm{kg})$ to block $D_{2}$ receptors. The rats were then challenged with nicotine $1 \mathrm{~h}$ later, when the haloperidol effect was maximal, and $4 \mathrm{~h}$ later, when the haloperidol effect was over.

\section{Alert rats}

We prepared two rats for chronic stimulation and recording. We anesthetized the rats with ketamine $(90 \mathrm{mg} / \mathrm{kg})$ and xylazine $(10 \mathrm{mg} / \mathrm{kg}$ ). Using aseptic procedures, we implanted a nerve cuff (Teflon tubing with a pair of stainless steel wires) around the $\mathrm{SO}$ and OOemg electrodes into the orbicularis oculi muscle near the anterior and posterior aspects of the upper eyelid. These wires were led subcutaneously to the female end of a connector embedded in a dental acrylic mound attached to the skull. A silver wire placed on the skull provided a ground. Four to six self-tapping, stainless steel, screws [1-72, $3 / 16$ in. $(4.763 \mathrm{~mm})$, Small Parts MX-172-3] were screwed into the skull to anchor a dental acrylic base. The screws were covered with dental acrylic, which served as a base for the stimulating and recording leads. The rats recovered for 2 days or longer before testing began.

A lightweight lead plugged into the connector on the dental acrylic mound. This connection allowed stimulation through the 
nerve cuff and recording of the OOemg while the rat moved freely around the cage. Blinks were elicited every $20 \pm 3 \mathrm{~s}$. The rats showed no signs of discomfort during this procedure. To demonstrate that activation of dopamine receptors could mimic the effect of nicotine on the blink reflex, rats received apomorphine hydrochloride ( $4 \mathrm{mg} / \mathrm{kg}$ subcutaneously; Sigma, A-4393) after 15 control blinks were recorded. Fifteen reflex blinks were then recorded every $10 \mathrm{~min}$ for $1 \mathrm{~h}$.

\section{Drugs}

For the anesthetized-rat experiments, we utilized nicotine [(-)nicotine di-(+)-tartate salt, Sigma, N-5260] mixed with saline to form concentrations of $0.25-2.5 \mathrm{mg} / \mathrm{cc}$. Haloperidol (Research Biochemicals, $\mathrm{H}-100$ ) was mixed with $1 \%$ ascorbic acid to a concentration of $0.2 \mathrm{mg} / \mathrm{cc}$. For the alert-rat studies, apomorphine was mixed in saline to a concentration of $4 \mathrm{mg} / \mathrm{cc}$. All drugs were prepared on the day of the experiment.

\section{Data collection and analysis}

For both the human and the rats, data were collected and stored online on a personal computer. In the human study, lid and OOemg were sampled at $5000 \mathrm{~Hz}$. The computer rectified the OOemg record and integrated the muscle activity. The records could be analyzed individually or averaged (Evinger et al. 1991). In rats, we sampled the OOemg at $3000 \mathrm{~Hz}$, but did not measure lid position. OOemg records were rectified and integrated over periods specified by the investigator for each series of trials. The data was analyzed off-line for latency and OOemg magnitude. For both species, OOemg responses were filtered (bandpass $-3 \mathrm{~dB}$ points at 300 and $5000 \mathrm{~Hz}$ ) and collected with 12-bit accuracy (Data Translation DT2801-A).

\section{Results}

One advantage offered by the blink reflex is the extraordinary consistency and reliability of the response both within and between subjects. For example, stimulating the $\mathrm{SO}$ in alert rats, prepared for chronic stimulation and recording, elicits virtually identical OOemg responses day after day (Fig. 1). The fact that the standard errors of the mean are smaller than the symbols in Figs. 3, 5, 7, 9, and 11 further emphasizes the small within-subject variability. Between subjects, variability is also remarkably small. For example, the response latency of the SO-elicited blink among 83 human subjects had less than $10 \%$ variability (Kimura 1989). Similarly, the entire range of latencies for corneally elicited blinks was only $11.8-13.6 \mathrm{~ms}$ for all our anesthetized rat subjects. Thus, the consistency and dependability of the blink reflex allowed us to reveal changes reliably with small numbers of subjects.

\section{Effect of nicotine on the blink reflex}

Chewing nicotine gum. Rapidly chewing $10 \mathrm{mg}$ of nicotine in gum decreased blink magnitude and increased blink latency for a normal human (Fig. 2). Just as occurs with cigarette smoking (Evinger et al. 1988), nicotine decreased the magnitude of all OOemg components $(t=4.97, d f=14$, $P<0.001)$ and the ensuring blink, but increased the latency of the R2 component of the OOemg $(t=3.09$,

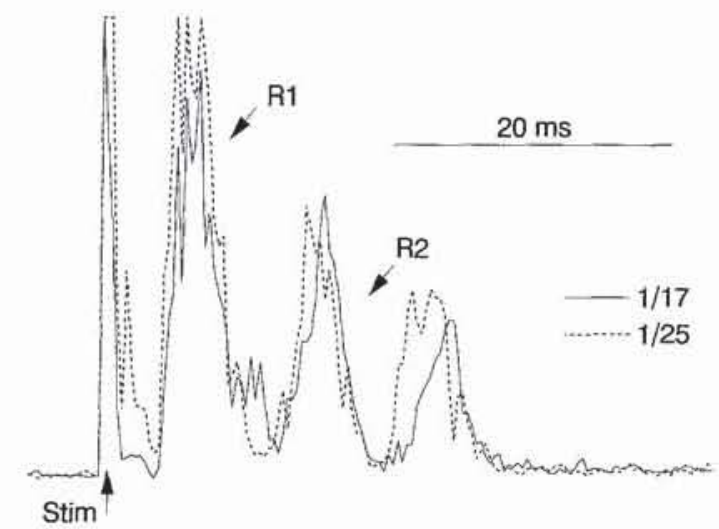

Fig. 1. Blinks elicited by stimulation of the supraorbital branch of the trigeminal nerve (SO) in an alert rat. Each trace is the averaged, rectified orbicularis oculi electromyogram (OOemg) response to 15 identical SO stimuli. The response shown by the solid line was collected 1 week prior to the response shown by the dashed line. $R 1$, short-latency response; $R 2$, long-latency response; Stim, stimulus artifact

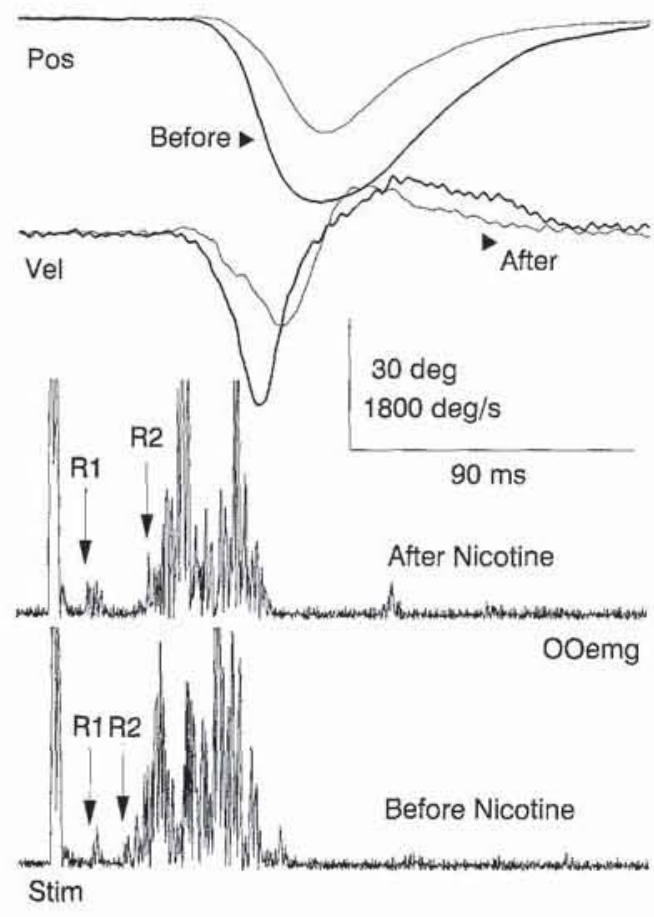

Fig. 2. A single reflex blink of a human subject elicited by stimulation of the SO nerve before (thick lines) and after (thin lines) chewing $10 \mathrm{mg}$ of nicotine gum. OOemg, rectified orbicularis oculi EMG activity; Pos, upper eyelid position; Stim, stimulus artifact; Vel, lid velocity

$d f=14, P<0.01)$ without altering R1 latency. Unlike the transient effect of cigarette smoking on the blink reflex, however, nicotine gum influenced the blink throughout the test period (Fig. 3). The longer lasting effect of nicotine gum probably resulted from the slower kinetics of nicotine absorption with nicotine gum than with cigarette smoking (Fagerström 1989; Russell et al. 1983). It is unlikely that 
the $10 \mathrm{mg}$ dose of nicotine gum was responsible for the longer lasting effect because chewing $10 \mathrm{mg}$ of nicotine gum yields blood levels of nicotine only slightly higher than those associated with cigarette smoking (Fagerström 1989). Thus, in humans, nicotine appears to be the agent in cigarette smoke responsible for modifying the blink reflex.

Intravenous injection of nicotine. Intravenous injection of nicotine into urethane-anesthetized rats decreased the OOemg amplitude of corneally elicited blinks and increased OOemg latency (Fig. 4). Both rats and humans

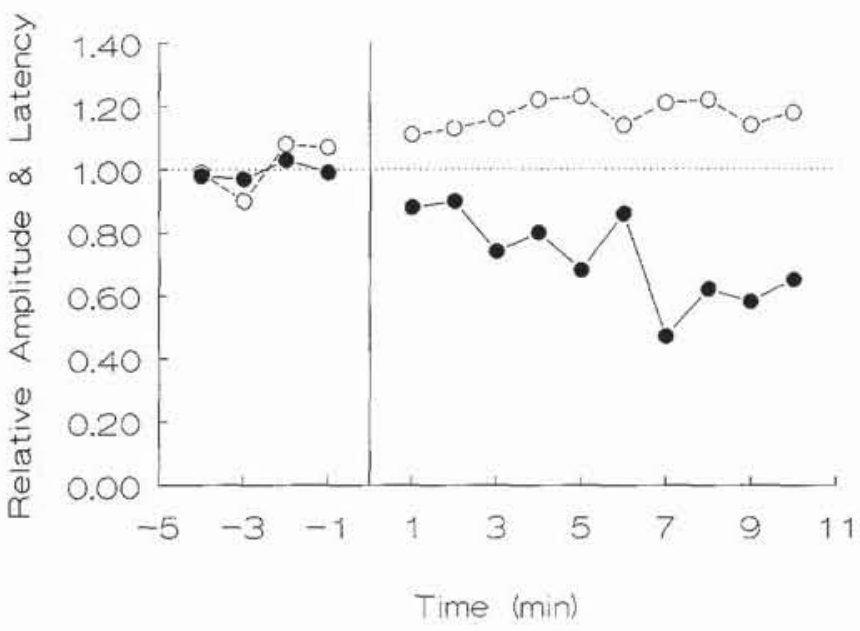

Fig. 3. R2 amplitude ( $\bullet$ ) and latency (o) relative to mean prenicotine values (dotted line) with supraorbital nerve stimulation before and after chewing $10 \mathrm{mg}$ nicotine in gum for one human. Each point is the mean of three blinks

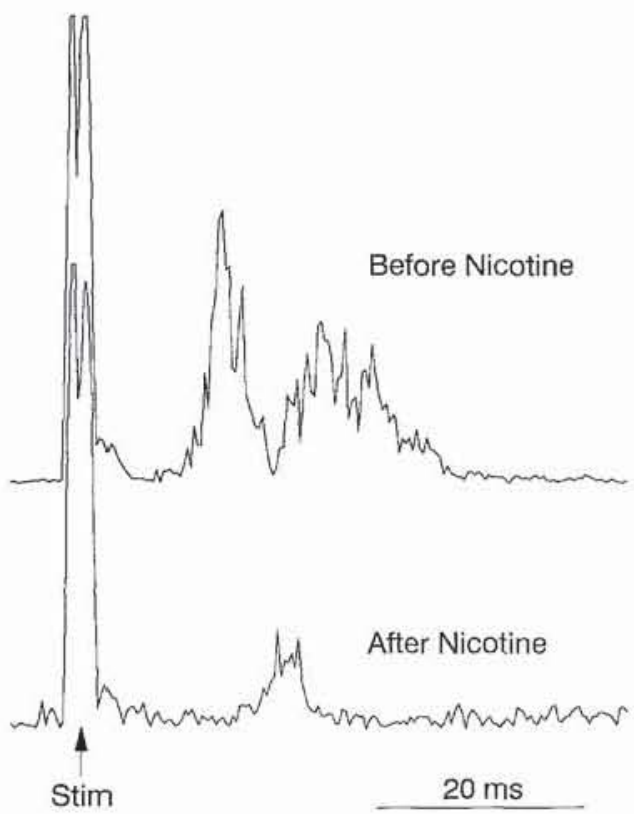

Fig. 4. Averaged ( 5 trials), rectified OOemg responses elicited by corneal stimulation (Stim) before and after intravenous nicotine injection $(1.3 \mathrm{mg} / \mathrm{kg})$ in a urethane-anesthetized rat exhibit only a single component of OOemg activity with blinks elicited by stimulation of the cornea (Figs. 4, 6; Accornero et al. 1980; Beradelli et al. 1985), in contrast to activity shown with SO-elicited blinks. In some rats, the OOemg response evoked by corneal stimulation appeared similar to the $\mathrm{R} 1$ and $\mathrm{R} 2$ responses elicited by $\mathrm{SO}$ stimulation in the alert rat (e.g., Fig. 4). More often, however, rats exhibited a single burst of OOemg response with corneal stimulation (Fig. 6). The corneally elicited and SO-elicited blinks exhibited very different physiological properties. For example, it was impossible to elicit an R1 and R2 response with $\mathrm{SO}$ stimulation in urethane-anesthetized rats, even though corneally elicited blinks were robust. Nevertheless, the latency of the R2 component of SOelicited blinks (Fig. 12) and the latency of the corneally elicited OOemg activity (Figs. 4, 6) were similar.

Nicotine doses equal to or above $0.32 \mathrm{mg} / \mathrm{kg}$ altered both blink amplitude and latency (Fig. 5). Doses of nicotine larger than $0.32 \mathrm{mg} / \mathrm{kg}$ failed to increase the magnitude of the effect on the blink reflex. Thus, doses equal to or greater than $0.32 \mathrm{mg} / \mathrm{kg}$ elicited the maximal effect of nicotine in the anesthetized rat. The increase in latency and decrease in OOemg amplitude were statistically significant for all animals ( $P<0.001$ for all $t$ tests). At all three nicotine doses tested, the injection completely suppressed the blink reflex for 1-3 min and reduced the amplitude for another 3-7 min. Blink latency tended to recover more quickly then blink amplitude. For example, the OOemg amplitude of the rat receiving $1.3 \mathrm{mg} / \mathrm{kg}$ nicotine returned to prenicotine levels approximately $8 \mathrm{~min}$ after the nicotine injection, but blink latency recovered in less than $5 \mathrm{~min}$. This observation suggests that nicotine differentially affects blink amplitude and latency. Consistent with this conclusion, nicotine doses of $0.25 \mathrm{mg} / \mathrm{kg}$ produced a strong suppression of blink amplitude, but little or no increase in OOemg latency (Fig. 6).

Decerebration. Nicotine could act directly on the pontinemedullary neurons subserving the blink reflex, or nicotine

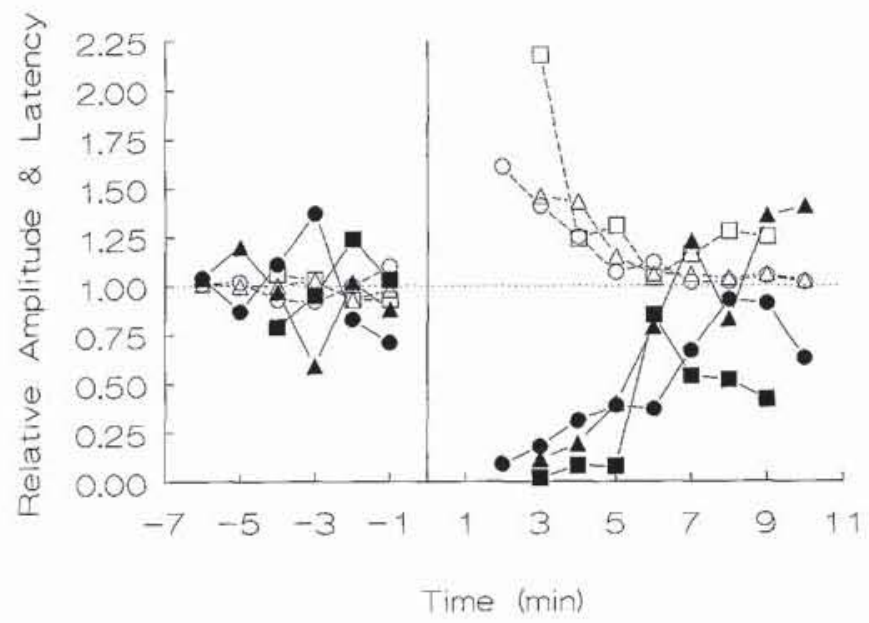

Fig. 5. OOemg amplitude (filled symbols) and latency (open symbols) relative to mean prenicotine values (dotted line) in rats receiving 0.32 $(\mathbf{E}, \square), 1.3(\bullet, 0)$, or $2.5(\boldsymbol{\Delta}, \Delta) \mathrm{mg} / \mathrm{kg}$ nicotine. Each point is the mean of three blinks 


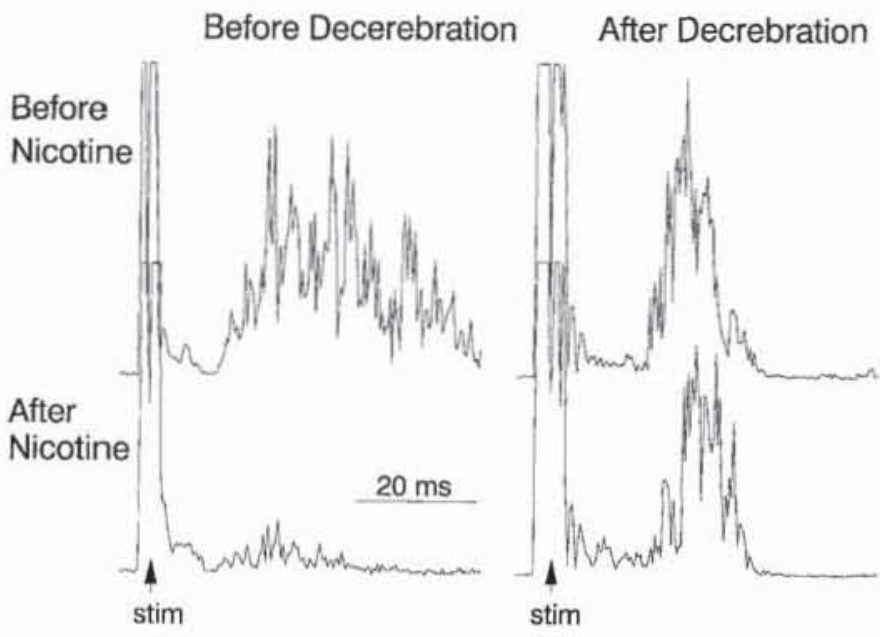

Fig. 6. Averaged ( 5 trials), rectified OOemg from corneal stimulation (Stim) before and after $0.25 \mathrm{mg} / \mathrm{kg}$ nicotine, showing the effect of decerebration

could modify descending neurons that modulate the blink reflex. We eliminated descending influences by testing the effect of nicotine on the blink reflex following precollicular decerebration. Decerebration abolished the actions of intravenously injected nicotine without eliminating the blink reflex (Figs. 6, 7). Prior to decerebration, the decrease in OOemg amplitude following a nicotine injection was statistically significant $(t=2.83, d f=14, P<0.025)$, but it was not significant after decerebration $(t=0.4$, $d f=14, P=$ n.s.). Thus, nicotine must modulate the blink reflex by acting primarily on circuits rostral to the decerebration. Since the striatum is rich in nicotinic receptors and basal ganglia disorders modify the blink reflex, nicotine could modify blinking through the basal ganglia.

\section{Effect of substantia nigra stimulation on the blink reflex}

In two urethane-anesthetized rats, electrical stimulation in the region of the substantia nigra mimicked the effect of nicotine injections on the blink reflex (Figs. 8, 9). Stimulating the cornea once every $10 \mathrm{~s}$ produced modest habituation of the blink reflex. Preceding corneal stimulation by $7 \mathrm{~s}$ of $10-\mathrm{Hz}, 40-\mu \mathrm{A}, 60-\mu \mathrm{s}$ stimulation on the dorsal edge of the substantia nigra reticulata, however, decreased OOemg amplitude, increased the rate of habituation, and increased OOemg latency (Figs. 8,9 ) relative to corneal stimulation alone (Figs. 8,9; OOemg amplitude: $t=2.74$, $d f=13, P<0.025$; latency: $t=5.34, d f=13, P<0.001)$. Deterioration in the condition of the animal or the responsiveness of trigeminal pathways did not cause this modification of the blink reflex. Forty minutes after combined nigral and corneal stimulation, electrical stimulation of the cornea alone elicited blinks of normal amplitude and latency relative to the blinks evoked prior to nigral stimulation (Fig. 9). These data are consistent with the suggestion that nicotine acts through the basal ganglia to decrease blink amplitude and increase blink latency. If this hypothesis is correct, blocking dopamine receptors should

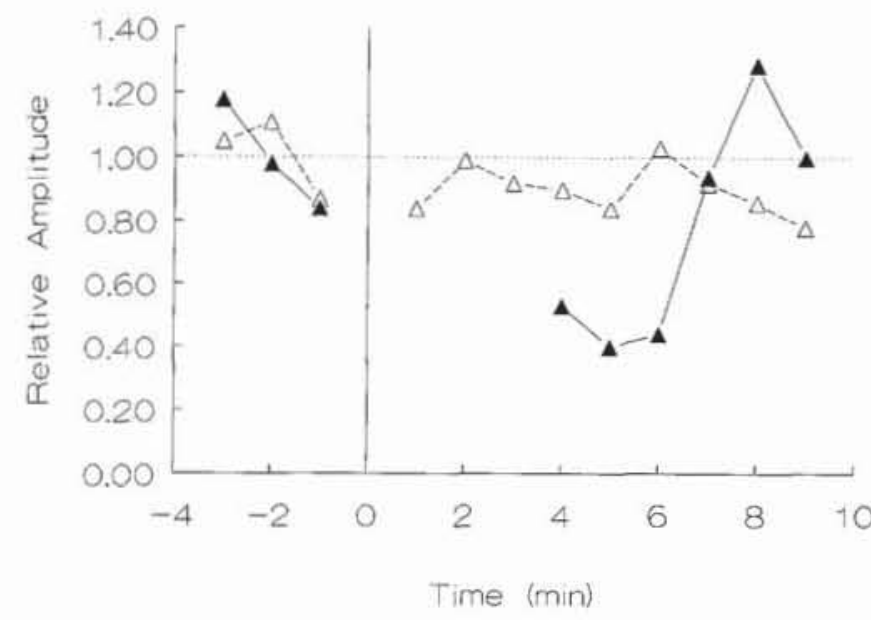

Fig. 7. OOemg amplitude relative to mean prenicotine values (dotted line) before and after $0.25 \mathrm{mg} / \mathrm{kg}$ nicotine, before $(\boldsymbol{\Delta})$ and after $(\Delta)$ decerebration. Each point is the mean of three blinks

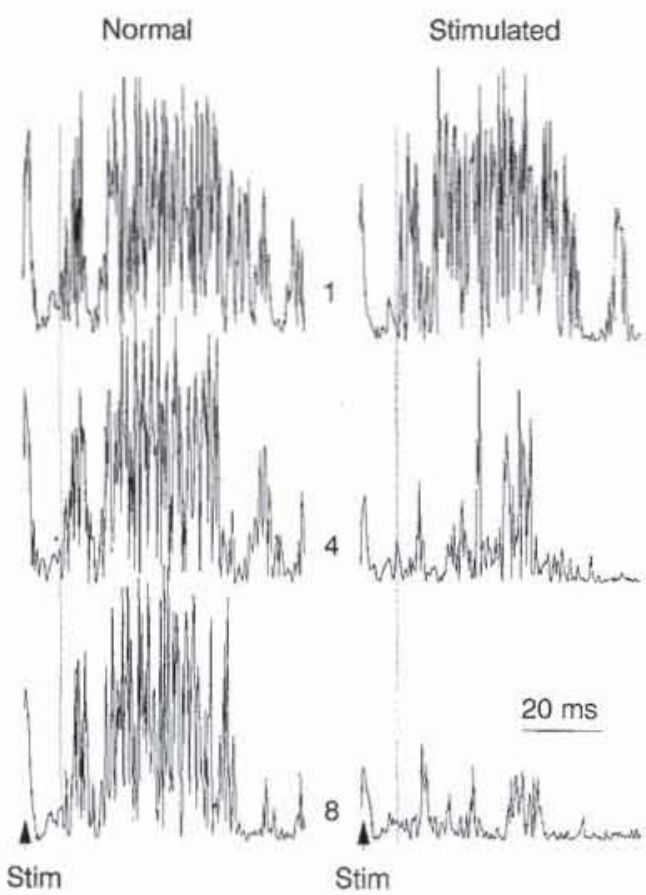

Fig. 8. Rectified OOemg of trials 1, 4, and 8 elicited by corneal stimulation (Stim). Left, normal. Right, corneal stimulation preceded by nigral stimulation. Dashed lines indicate start of OOemg activity on trial $l$

prevent nicotine's modulation of the blink reflex because nicotine causes the release of dopamine from presynaptic terminals in the striatum (Giorguieff-Chesselet et al. 1979; Sakurai et al. 1982; Schultz and Zigmond 1989).

\section{Pharmacological manipulation of the dopamine receptors}

Effect of haloperidol on anesthetized rats. We investigated the role of $\mathrm{D}_{2}$ receptors in modulating the blink reflex by 
examining the effect of nicotine on the blink reflex before and after administration of haloperidol, a $\mathrm{D}_{2}$ receptor antagonist (Figs. 10, 11). Before haloperidol administration, nicotine injection caused the expected decrease in OOemg magnitude with both corneal- (Fig. $11 ; t=6.53$, df $=11, P<0.001$ ) and air-puff-elicited (Figs. 10, 11; $t=2.54$, $d f=11, \quad P<0.025)$ blinks. Injection of haloperidol $(0.15 \mathrm{mg} / \mathrm{kg}$ i.p.), however, reduced or abolished nicotine's modification of the blink reflex (Figs. 10, 11; cornea: $t$ $=1.39, d f=14, P=$ n.s.; air puff: $t=1.5, d f=14, P=$ n.s.). Thus, nicotine appears to modulate the blink reflex via dopamine receptors.

Given that blockade of $D_{2}$ receptors prevents nicotine's modification of the blink reflex, activation of $\mathrm{D}_{2}$ receptors should mimic nicotine's effect on the blink reflex. We investigated the effect of apomorphine, a $\mathrm{D}_{2}$ agonist, on OOemg activity elicited by SO stimulation in chronic-

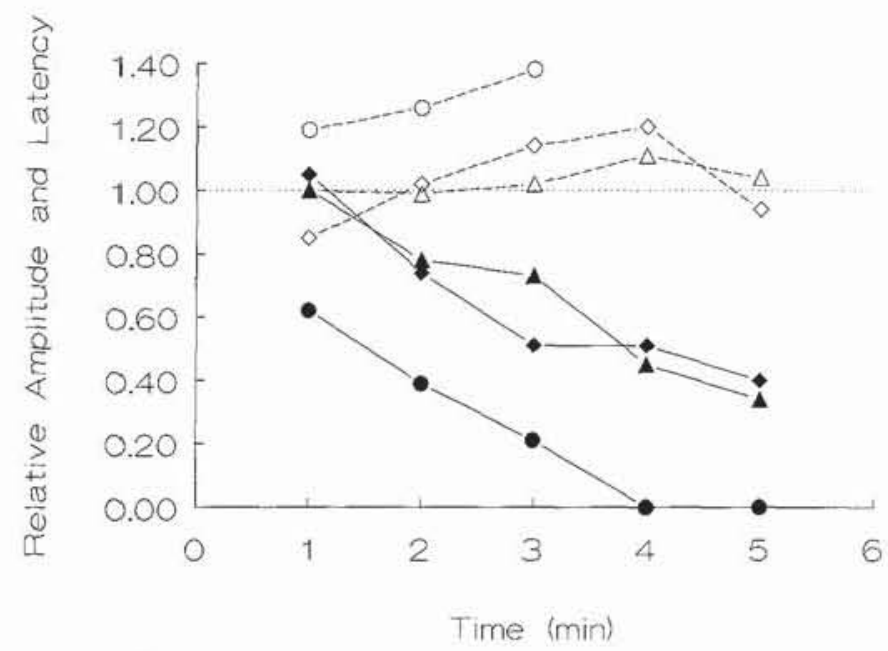

Fig. 9. OOemg amplitude (filled symbols) and latency (open symbols) relative to first normal reflex blink $(\boldsymbol{\Lambda}, \Delta)$. $(\bullet, \circ$,$) blinks preceded by$ nigral stimulation; $(\bullet, \diamond$,$) recovery. Each point is the mean of three$ blinks

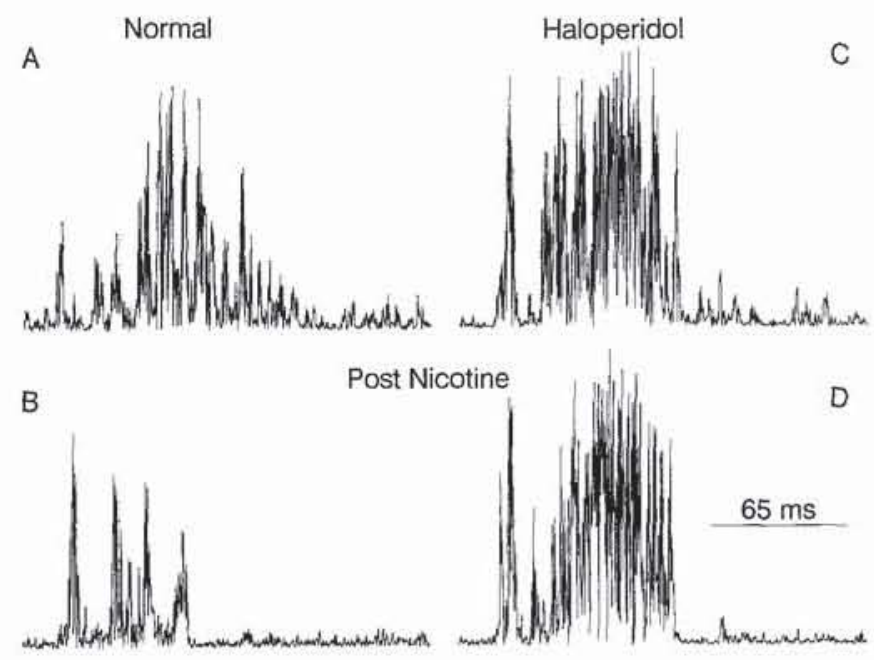

Fig. 10. A-D Rectified OOemg of individual blinks elicited by an airpuff stimulus before (A, C) and after (B, D) $0.25 \mathrm{mg} / \mathrm{kg}$ nicotine. A, B Before haloperidol. C, D One hour after $0.15 \mathrm{mg} / \mathrm{kg}$ haloperidol

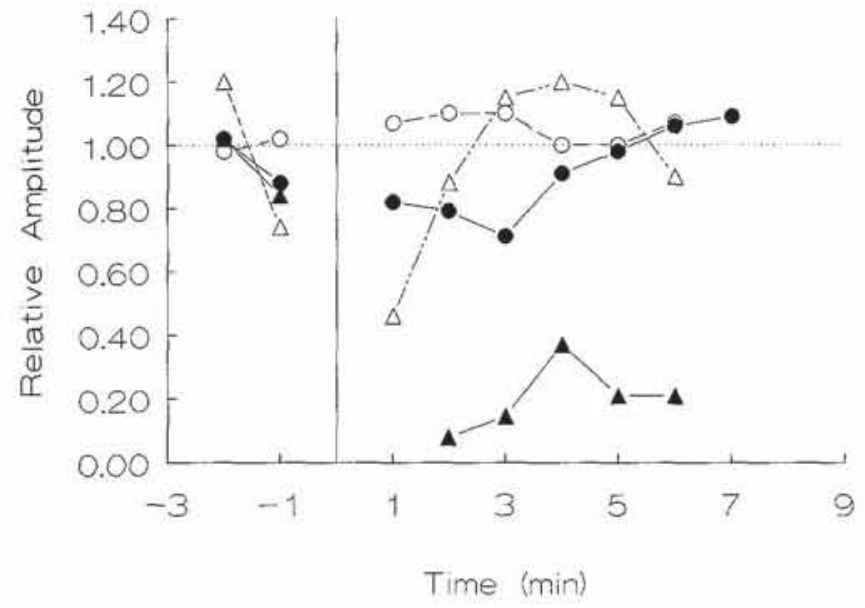

Fig. 11. OOemg amplitude of air-puff (open symbols) and corneal (filled symbols)-elicited blinks relative to mean prenicotine value (dotted line) before $(\boldsymbol{\Lambda}, \Delta)$ and $1 \mathrm{~h}$ after $(\bullet, 0) 1.5 \mathrm{mg} / \mathrm{kg}$ haloperidol

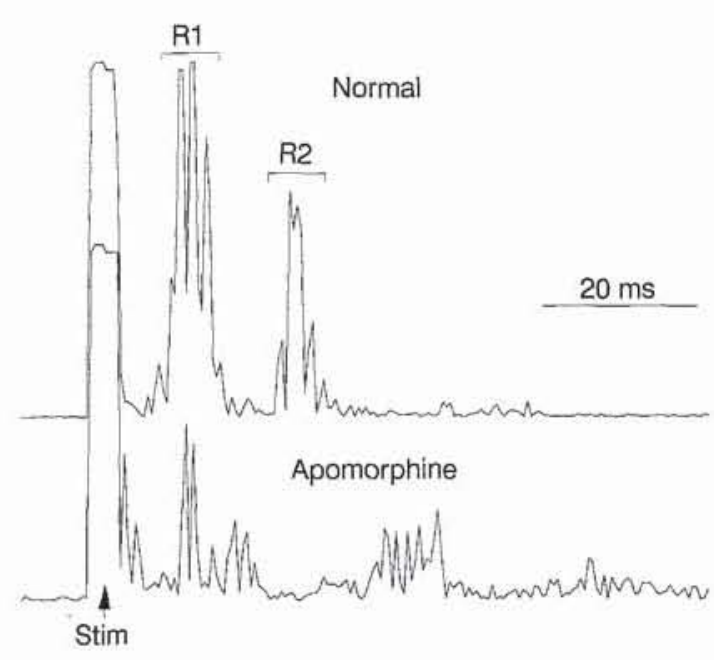

Fig.12. Individual, rectified OOemg responses to supraorbital nerve stimulation in an alert rat before (top trace) and after (bottom trace) 4 $\mathrm{mg} / \mathrm{kg}$ apomorphine

ally prepared, alert rats (Fig. 12). Injection of $4 \mathrm{mg} / \mathrm{kg}$ apomorphine produced a decrease in both $\mathrm{R} 1$ and $\mathrm{R} 2$ amplitude of the SO-elicited evoked blink ( $t=4.47$, $d f=16, P<0.001$ ), but primarily increased R2 latency $(t=5.49, d f=11, P<0.001 ;$ Fig. 12). Since this dose of apomorphine caused the rats to chew incessantly, OOemg records become noisy, and accurate measurements of latency and amplitude were possible only on 10-15 selected records for each rat. Of the two rats tested, one showed no change in R1 latency following apomorphine, while the second exhibited an insignificant, 1-ms increase in R1 latency. In contrast, R2 latency increased by $7.2 \mathrm{~ms}$ in the first rat and $5.4 \mathrm{~ms}$ in the second. OOemg amplitude of the two blink components decreased by $31 \%$ in the first rat and $41 \%$ in the second rat. The effect lasted for the entire hour following the apomorphine injection. This result is qualitatively identical to that obtained with nicotine chewing gum. Thus, activation of $\mathrm{D}_{2}$ receptors mimics the effects of nicotine on the blink reflex. 


\section{Discussion}

\section{Comparison of rat and human reflex blinks}

The reflex blinks of rats and humans are very similar. In both species, stimulation of the SO elicits a pair of OOemg responses, R1 and R2 (Figs. 1, 12; Kimura 1989; Shahani and Young 1973, for review). R1, which shows little intersubject variability, probably arises from a threeneuron arc. The R2 latency varies widely among subjects and clearly arises from an oligosynaptic circuit (Ongerboer de Visser 1983). The rapid activation of the rat OOemg from SO stimulation $(4-7 \mathrm{~ms})$ strongly suggests that rat R1 also arises from no more than a trisynaptic circuit. As in humans, the rat R2 latency of $14-20 \mathrm{~ms}$ is consistent with an oligosynaptic circuit. In the two species, corneal stimulation usually elicits a single burst of OOemg activity (Fig. 4; Accornero et al. 1980; Beradelli et al. 1985). The observation that corneal blinks and the R2 response have a similar latency led to the proposal that R2 and corneal blinks originate from the same neural circuit (e.g., Rushworth 1962; Kugelberg 1952). Recent human studies, however, demonstrated that the two types of blink could not utilize identical circuitry (Beradelli et al. 1985; Cruccu et al. 1986). Similarly, the fact that there was differential modification of the corneal blink reflex and the $\mathrm{R} 2$ response in two cases shows that the same neural circuit also does not subserve the two responses in the rat. First, although similar to R2, the corneal blink has a distinctly shorter latency than the R2 component (Figs. 1, 4,12 ). Second, in urethane-anesthetized rats, the corneal reflex is brisk, but SO-evoked blinks never have an R2 component. Finally, the regions of the spinal trigeminal nucleus receiving primary afferents from the cornea and the SO are different (Marfurt and Del Toro 1987; Pellegrini and Evinger 1991). Nevertheless, the corneal blink and the $\mathrm{R} 2$ response could share some interneurons, since nicotine affects the two blinks similarly in the rat.

Despite the comparable neural circuitry underlying blinks in rats and humans, two differences exist. First, the rat blink reflex is not consensual, while both the human corneal blink reflex and the human R2 response with SO stimulation are bilateral. In alert rats, stimulation of the SO appears only to activate the contralateral OOemg when the rat is startled. In urethane-anesthetized rats, unilateral corneal stimulation never elicits a contralateral reaction. Nevertheless, both rat and human $\mathrm{R} 1$ responses are normally ipsilateral. Second, the rat R 1 component is larger and has a lower threshold than the R2 component. The opposite is true for humans (e.g., Sanes and Ison 1979). This second difference may reflect the evolutionary significance placed on the $\mathrm{R} 1$ and $\mathrm{R} 2$ responses in frontaland lateral-eyed species.

\section{Nicotine-induced modification of the blink reflex}

Cigarette smoking transiently decreases blink magnitude and increases R2 latency (Evinger et al. 1988). While other elements of cigarette smoke could potentially modify the blink reflex, nicotine appears to be the responsible agent.
Nicotine gum produces the same alterations in the blink reflex as tobacco smoking but over a longer time course. This difference may follow from the prolonged release of nicotine with nicotine gum and the slowness of gastrointestinal uptake of nicotine compared with the rapid passage of nicotine into the bloodstream through the lung and nasal passages with smoking (Fagerström 1989; Roughton 1945; Russell et al. 1980, 1983). Intravenous injection of nicotine in rats, however, produced the same modifications as cigarette smoking and matched the time course engendered with smoking. Thus, nicotine must be the element in cigarette smoke responsible for modifying the blink reflex as well as eye movements (Sibony et al. 1990).

Nicotine could act directly on blink reflex neurons in the lower brainstem with nicotinic receptors, or nicotine could activate a descending system impinging on blink circuits to modify the blink reflex indirectly. The fact that nicotine only modifies the latency of one component of the blink reflex and that decerebration eliminates the effects of nicotine demonstrates that nicotine's main actions are central rather than on the orbicularis oculi muscle. Since precollicular decerebration eliminates the nicotine effect on the blink reflex, nicotine is unlikely to be acting exclusively on blink reflex interneurons, all of which lie caudal to the pretectum (Ongerboer de Visser 1983). The ability of a dopaminergic receptor antagonist, haloperidol, to block nicotine's effect implies that nicotine acts indirectly on the blink reflex by causing the release of dopamine. In addition to blocking dopamine receptors, haloperidol also exhibits some anticholinergic properties. This characteristic might explain some of haloperidol's elimination of nicotine's affect on the blink reflex. Nevertheless, the fact that apomorphine, a $\mathrm{D}_{2}$ agonist, mimicked nicotine's effects supports the proposal that nicotine predominantily exerts its action on the blink reflex via the release of dopamine. These observations implicate the basal ganglia in nicotine's effects on the blink reflex.

Dopaminergic neurons of the basal ganglia are rich in nicotinic receptors. Dopaminergic neurons of the substantia nigra compacta contain nicotinic receptors on their terminals in the striatum, as well as on their somata (Abood et al. 1981; Bolam et al. 1991; Clarke 1989; Clarke and Pert 1985; Hunt and Schmidt 1978; Semba and Fibiger 1989). Intravenous and subcutaneous injection of nicotine activates dopaminergic neurons in a dose-dependent fashion (Clarke et al. 1985; Lichtensteiger et al. 1982; Westfall et al. 1989), with a maximum increase in firing frequency caused by $0.5 \mathrm{mg} / \mathrm{kg}$ nicotine administered intravenously (Mereu et al. 1987). Nicotine causes the release of dopamine in the striatum (GiorguieffChesselet et al. 1979; Sakurai et al. 1982; Schultz and Zigmond 1989). Thus, in our experiments, nicotine should have markedly increased the release of dopamine in the striatum. Moreover, since dopamine receptor blockade obstructed nicotine's effect on the blink reflex, the increased release of dopamine appears to be a crucial step in modifying the blink reflex.

Clinical studies demonstrate that abnormally low levels of dopamine in the striatum disrupt normal blink reflexes. Advanced stages of Parkinson's disease result in 
an increased excitability of both the R1 and the R2 components of the blink reflex (Klawans and Goodwin 1969; Messina et al. 1972; Pearce et al. 1969; Rushworth 1962). Reduction in dopamine levels through treatment with haloperidol or reserpine also increase the excitability of the blink reflex (Boiardi et al. 1975; Ferguson et al. 1978; Raffaele et al. 1988). Similarly, reduction of dopamine in rats by injection of 6-hydroxydopamine elevates blink reflex excitability (Basso et al. 1991). These observations suggest that increased levels of dopamine in the striatum, such as those induced by nicotine should decrease the excitability of the blink reflex.

The basal ganglia can influence interneurons involved in the blink reflex by modifying descending cortical or superior colliculus projections. Early studies of the effect of Parkinson's disease on the blink reflex suggested that cortical involvement accounted for the increased excitability (Klawans and Goodwin 1969; Pearce et al. 1969). Recent studies in primates with 1-methyl-4-phenyl-1,2,3,6tetrahydropyridine- induced Parkinson's disease reveal an increased inhibition of thalamic neurons (e.g. DeLong 1990), which should result in reduced cortical output. Similarly, the superior colliculus should receive increased inhibition in Parkinson's disease. Consistent with this result, injection of the gamma-aminobutyric acid agonist, muscimol, into the superior colliculus produces an increase in blink reflex excitability similar to that present in rats with Parkinsonian-like symptoms (Basso et al. 1991; Basso and Evinger 1992). Therefore, descending cortical and collicular neurons must modulate a network that inhibits blink reflex interneurons. In this scheme, increasing striatal dopamine levels with nicotine would increase inhibition of blink reflex interneurons and decrease the magnitude of the blink reflex.

Acknowledgements. We would like to thank Donna Malysz for technical assistance. This work was supported by NEI grant EY07391 (CE), a Parkinson Disease Foundation summer fellowship (MAB), and a NATO fellowship from the DAAD (AKEH).

\section{References}

Abood LG, Reynolds DT, Booth H, Bidlack JM (1981) Sites and mechanisms for nicotine's actions in the brain. Neurosci Biobehav Rev 5: 479-486

Accornero N, Beradelli A, Bini G, Cruccu G, Manfredi M (1980) Corneal reflex elicited by electrical stimulation of the human cornea. Neurology 30: 782-785

Araujo DM, Lapchak PA, Collier B, Quirion R (1988) Characterization of $N-\left[{ }^{3} \mathrm{H}\right]$ methylcarbamylcholine binding sites and effect of $N$-methylcarbamylcholine on acetylcholine release in rat brain. I Neurochem 51: 292-299

Basso MA, Evinger C, (1992) Exciting the blink through inhibition. Basal ganglia control of the blink reflex. Soc Neurosci Abstr 18: 856

Basso MA, Evinger C, Strecker RE (1991) Nigrostriatal lesions modulate the blink reflex. Soc Neurosci Abstr 17: 461

Benowitz NL, Porchet H, Jacob P III (1989) Nicotine dependence and tolerance in man: pharmacokinetics and pharmacodynamic investigations. Prog Brain Res 79: 279-288

Beradelli A, Cruccu G, Manfredi M, Rothwell JC, Day BL, Marsden CD (1985) The corneal reflex and the R2 component of the blink reflex. Neurology 35: 797-801
Boiardi A, Bussone G, Caccia MR, Rocca E (1975) Electrophysiological evidence for a neurohormonal dependence in the changes of the late glabellar response in man. Eur Neurol 13: 513-518

Bolam JP, Francis CM, Henderson Z, (1991) Cholinergic input to dopaminergic neurons in the substantia nigra: a double immunocytochemical study. Neuroscience 41: 483-494

Bollen E, Arts RJHM, Roos RAC, Van Der Velde EA, Buruma OJS (1986) Brainstem reflexes and brainstem auditory evoked responses in Huntington's chorea. J Neurol Neurosurg Psychiatry 49: $313-315$

Caraceni T, Avanzini G, Spreafico R, Negri S, Broggi G, Girotti F (1976) Study of the excitability cycle of the blink reflex in Huntington's chorea. Eur Neurol 14: 465-472

Clarke PBS (1989) Mapping of nicotinic receptors by autoradiographic techniques and the effect of experimental lesions. Prog Brain Res 79: 65-71

Clarke PBS, Pert A (1985) Autoradiographic evidence for nicotine receptors on nigrostriatal and mesolimbic dopaminergic neurons. Brain Res 348: $355-358$

Clarke PBS, Hommer DW, Pert A, Skirboll LR (1985) Electrophysiological actions of nicotine on substantia nigra single units. Br J Pharmacol 85: 827-835

Cruccu G, Agostino R, Berardelli A, Manfredi M (1986) Excitability of the corneal reflex in man. Neurosci Lett 63: 320-324

DeLong MR (1990) Primate models of movement disorders of basal ganglia origin. Trends Neurosci 13: 281-285

Esteban A, Mateo D, Giminez-Roldan S (1981) Early detection of Huntington's disease. Blink reflex and levodopa load in presymptomatic and incipient subjects. J Neurol Neurosurg Psychiatry $44: 43-48$

Evinger C, Sibony PA, Manning KA, Fiero RA (1988) A pharmacological distinction between the long and short latency pathways of the human blink reflex revealed with tobacco. Exp Brain Res 73: $477-480$

Evinger C, Manning KA, Sibony PA (1991) Eyelid movements. Mechanisms and normal data. Invest Ophthalmol 32: 387-400 Fagerström KO (1989) Treatment of nicotine dependence. Prog Brain Res 79: 321-328

Fagerström KO (1989) Treatment of nicotine dependence. Brain Res 79: $321-328$

Ferguson IT, Lenman JAR, Johnston BB (1978) Habituation of the orbicularis oculi reflex in dementia and dyskinetic states. $\mathrm{J}$ Neurol Neurosurg Psychiatry 41: 824-828

Giorguieff-Chesselet MF, Kemel ML, Wandscheer D, Glowinski J (1979) Regulation of dopamine release by presynaptic nicotinic receptors in rat striatal slices: effect of nicotine in low concentration. Life Sci 25: 1257-1262

Hunt S, Schmidt J (1978) Some observations on the binding patterns of $\alpha$-bungarotoxin in the central nervous system of the rat. Brain Res 157: 213-232

Kimura J (1989) Electrodiagnosis in diseases of nerve and muscle: principles and practice. Davis, Philadelphia.

Klawans HL and Goodwin JA (1969) Reversal of the glabella reflex in Parkinsonism by 1-DOPA. J Neurol Neurosurg Psychiatry 32: 423-427

Kugelberg E (1952) Facial reflexes. Brain 75: 385-396

Lichtensteiger W, Hefti F, Felix D, Huwyler T, Melamed E, Schlumpf M (1982) Stimulation of nigrostriatal dopamine neurones by nicotine. Neuropharmacology 21:963-968

Marfurt CF, Del Toro DR (1987) Corneal sensory pathway in the rat: a horseradish peroxidase tracing study. J Comp Neurol 261 $450-459$

Mereu G, Yoon K-WP, Boi V, Gessa GL, Naes L, Westfall TC (1987) Preferential stimulation of ventral tegmental area dopamine neurons by nicotine. Eur J Pharmacol 141: 395-399

Messina C, Di Rosa E, Tomasello F (1972) Habituation of blink reflexes in parkinsonian patients under levodopa and amantadine treatment. J Neurol Sci 40: 141-148

Ongerboer de Visser BW (1983) Anatomical and functional organization of reflexes involving the trigeminal system in man: jaw 
reflex, blink reflex, corneal reflex, and exteroceptive suppression. In: Desmedt JE (ed) Motor control mechanisms in health and disease. Raven Press, New York, pp 727-738

Pearce J, Aziz H, Gallagher JC (1969) Primitive reflex activity in primary and symptomatic Parkinsonism. J Neurol Neurosurg Psychiatry 31: 501-508

Pellegrini JJ, Evinger C (1991) Studies on the mechanism of blink reflex suppression. Soc Neurosci Abstr 17: 461

Raffacle R, Emery P, Palmeri A, Ricca G, Perciavalle V (1988) Influences of dopaminergic systems on the blink reflex. Ital J Neurol Sci 9: $351-354$

Roughton FJW (1945) The average time spent by blood in the human lung capillary and its relation to the rates of $\mathrm{CO}$ uptake and elimination in man. Am J Physiol 143: 621-633

Rushworth G (1962) Observations on blink reflex. J Neurol Neurosurg Psychiatry 25: 93-108

Russell MAH, Jarvis M, Iyer R, Feyerbrand C (1980) Relation of nicotine yield of cigarettes to blood nicotine concentrations in smokers. Br Med J 280: 972-976

Russell MAH, Feyerbrand C, Fernö O (1983) Nasal nicotine solution: a potential aid to giving up smoking. Br Med J 286: 683-684
Sakurai Y, Takano Y, Kohjimoto Y, Honda K, Kamiya H (1982) Enhancement of $\left[{ }^{3} \mathrm{H}\right]$ dopamine release and its $\left[{ }^{3} \mathrm{H}\right]$ metabolites in rat striatum by nicotinic drugs. Brain Res 242: 99-106

Sanes JN, Ison JR (1979) Conditioning auditory stimuli and the cutaneous eyeblink reflex in humans: differential effects according to oligosynaptic or polysynaptic central pathways. Electroencephalogr Clin Neurophysiol 47: 546-555

Schultz DW, Zigmond RE (1989) Neuronal bungarotoxin blocks the nicotinic stimulation of endogenous dopamine release from rat striatum. Neurosci Lett 98: 310-316

Semba K, Fibiger HC (1989) Organization of central cholinergic systems. Prog Brain Res 79: 37-64

Shahani BT, Young RR (1973) Blink reflexes in orbicularis oculi. In: Desmedt JE (ed) New developments in electromyography and clinical neurophysiology, vol III. Karger, Basel New York, pp 641-648

Sibony PA, Evinger C, Manning KA, Pellegrini JJ (1990) Nicotine and tobacco induced nystagmus (letter) Ann Neurol 28: 198

Westfall TC, Mereu G, Vickery L, Perry H, Naes L, Yoon K-WP (1989) Regulation of nicotine of midbrain dopamine neurons. Prog Brain Res 79: 173-186 